\begin{tabular}{|c|c|c|}
\hline $4 F$ & $\begin{array}{c}\text { European Association for the } \\
\text { Development of Renewable Energies, Environment } \\
\text { and Power Quality (EA4EPQ) }\end{array}$ & $\begin{array}{l}\text { International Conference on Renewable Energies and Power Quality } \\
\text { (ICREPQ'12) } \\
\text { Santiago de Compostela (Spain), 28th to 30th March, } 2012\end{array}$ \\
\hline
\end{tabular}

\title{
2D Analytical Calculation of the Open Circuit Electromagnetic Field Distribution in an Axial Flux Slotted Permanent Magnet Machine using Fourier Analysis.
}

\author{
J. M. Pérez García ${ }^{1}$ and F. A. Frechoso Escudero ${ }^{1}$ \\ ${ }^{1}$ Departament of Electrical Engineering \\ E.I.I., University of Valladolid \\ Escuela de Ingenierías Industriales, C/ Francisco Mendizábal, nº, 47014 Valladolid (Spain) \\ Phone number:+0034 9834236866 , Fax number +0034983423490, e-mail: julian@eii.uva.es, frechoso@eis.uva.es
}

\begin{abstract}
The paper presents an analytical method in order to obtain the electromagnetic field distribution in an axial flux slotted permanent magnet machine under no-load conditions. The method solves the Laplace equation in the gap of an slotted machine via magnetic scalar potential. Once the magnetic scalar potential is defined, the magnetic flux density components are computed.. Results issued from the proposed model are compared with those stemming from a 3D finite-element method (FEM) simulation. In addition to permanent magnet machines, this technique can be applied to any $2 \mathrm{D}$ geometry with the restriction that the geometry should consist of rectangular regions.
\end{abstract}

\section{Key words}

Analytical modelling, axial flux, Fourier analysis, magnetic scalar potential, permanent magnet machines.

\section{Introduction}

The axial flux permanent magnet (AFPM) machine is an attractive alternative to the radial flux permanent magnet machines due to its pancake shape, compact construction and high power density. AFPM machines are suitable for electrical vehicles, pumps, fans, etc. Since a large number of poles can be accommodated, this machines are ideal for low speed applications, as wind generators. The low speed generator does not require step-up gearbox in power transmission between the turbine and the generator, improving the reliability of the system and reducing the maintenance costs.

The knowledge of the flux density waveform is essential for the prediction of the motor performance. While numerical methods for field computation such as finiteelement method provide an accurate method to determine the flux density distributions in electrical machines, they are time-consuming and do not provide enough insight as compared with analytical solutions. Analytical methods allow to obtain the expression of the flux density waveform by means of solving the Laplace or Poisson equation in the studied region via the magnetic scalar potential ([1]-[4], [7]) or the magnetic vector potential ([5], [6], [8]-[10]), using current sheets to model the magnets and the armature current ([7], [8]), employing magnetic circuit theory ([9]) or applying conformal mapping ([9][11]). If the studied machine is an slotted one, the effects of the slots can be taken into account directly in the studied geometry ([4]-[6], [9]-[11]) or can be obtained starting from the expression of an slotless machine an introducing the Carter's coefficient ([1], [7]) or a permeance coefficient ([3]).

In this paper, we proposed a method which solves de Laplace's equation in the air gap of the machine via the scalar potential, using Fourier series and a set of boundary conditions, and taking into account the slotting of the machine. Once the magnetic scalar potential is defined, the magnetic flux density components are computed.

\section{Description of the method}

The method, which we call Fourier series method, was pointed by Hague [17] who calculated the magnetic field of linear currents in an air-gap with concentric circular cylindrical boundaries. Later, Nene [18] applies this technique to calculate the magnetic scalar potential distribution in rectangular regions with at least one known not null boundary condition. If there are known not null potential distributions on more than one boundary, the solution is obtained by applying the superposition theorem, by defining several simpler problems. With interconnected regions, the problem becomes increasingly difficult, although the technique is quite straightforward.

We applied this technique to an axial flux machine with three phases, eight poles and 24 slots. The inner rotor has 8 permanent magnets surface mounted, which are axially magnetised. The two outer slotted stator cores have an infinite permeability (which implies that the cores surface are equipotential). Its geometry is showed in the figure 1a, where $l_{m}$ is the magnet axial lenght, $L$ is the stator core axial lenght and $g$ is the gap thickness.

Looking radially inwards onto the machine and ignoring curvature, allows the machine to be represented as shown in figure $1 \mathrm{~b}$, where the $\mathrm{x}$-coordinate represents the 
circumferential direction and the y-coordinate the axial direction. This model assumes the radial direction to be infinite. By symmetry, only a half of the geometry is studied.

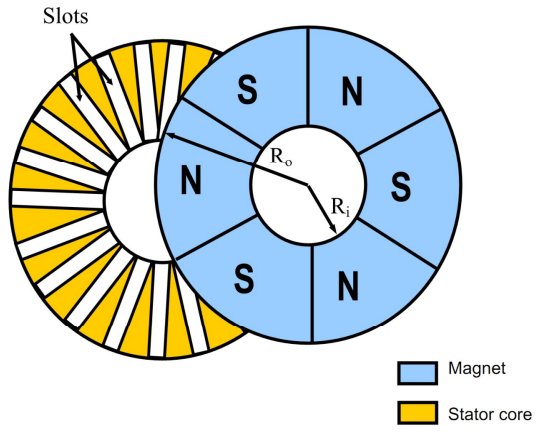

a)
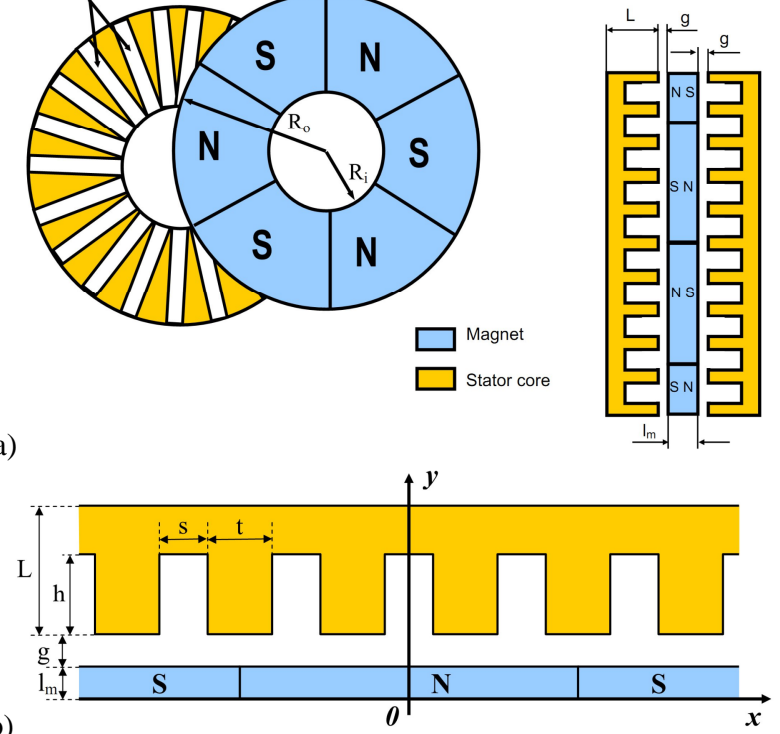

Fig. 1. Studied geometry. a) Three dimensions geometry. b) Simplified model in two dimensions.

We will apply the method of Fourier Series simplified geometry in two dimensions, in order to find the distribution of the scalar potential in the gap of the machine, considering the machine open circuit, ie, under no load conditions.

As discussed below, we need to know the value of the scalar magnetic potential on the surface of the magnets. To determine this value, we will study a slotless machine, in which the effect of the slots have been taken into account using the Carter's coefficient [1], [11], and then approach the study of the slotted machine. The Carter's coefficient is a factor that specifies how much an air gap must be increased analytically to take into account the presence of slots. [20] provides several analytical expressions for Carter's coefficient.

\section{Slotless machine}

Figure 2 shows the two dimensional geometry of the slotless machine. This two dimensional geometry can be reduced further by exploiting the symmetries than results from the repeating magnetic structure. Specifically, the field is symmetric about the vertical centre line of each pole and about the horizontal centre line of the magnets. The dashed line encloses this reduced geometry.

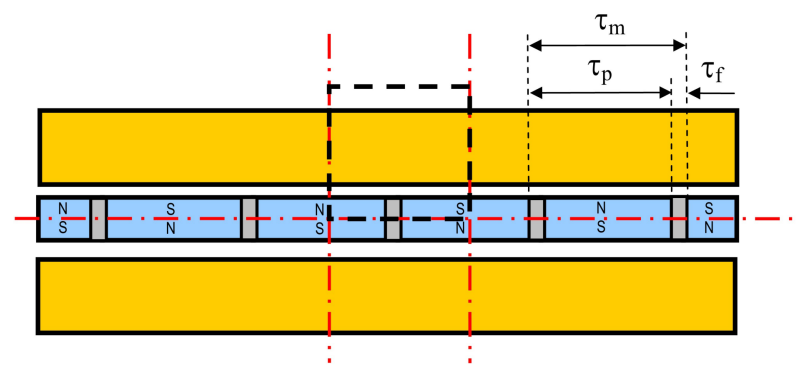

Fig. 2. Geometry of the slotless machine. The dashed line contains the reduced geometry.
Figure 3 shows the reduced geometry that reflects this geometry along with a cartesian reference frame that is used for the analysis below. In this figure, $\tau_{m}$ is the magnet width, $\tau_{f}$ the magnet spacer width and $\tau_{p}$ is the magnetic pole pitch.

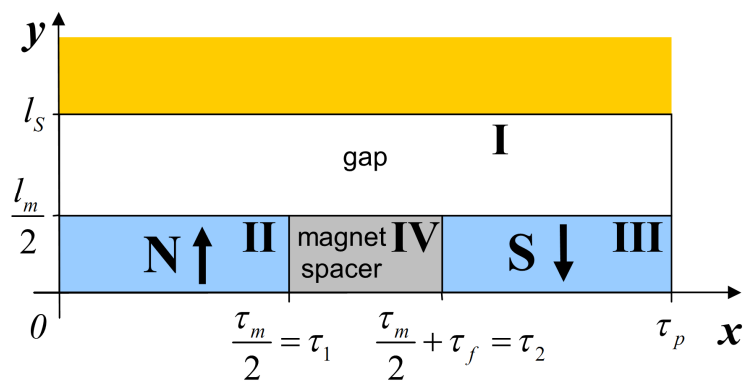

Fig. 3. Reduced two-dimensional geometry with reference frame.

The airgap/magnet regions are subdivided into four homogeneous sub-regions designated I, II, III and IV in figure 3. However, to simplify the analysis, sub-regions II, III and IV are combined by assuming that the magnet spacer is occupied by permanent magnet material but the magnet is fully magnetized only in sub-regions II and III and unmagnetized in sub-region IV. The corresponding cartesian component of magnetization, $M_{y}$, is shown in figure 4 and the new reduced geometry is shown in figure 5 .

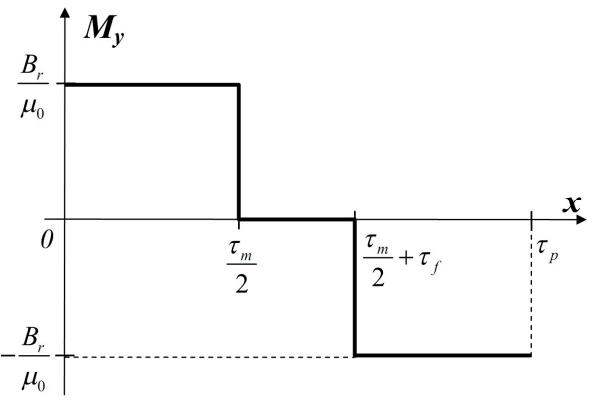

Fig. 4. Magnetization distribution.

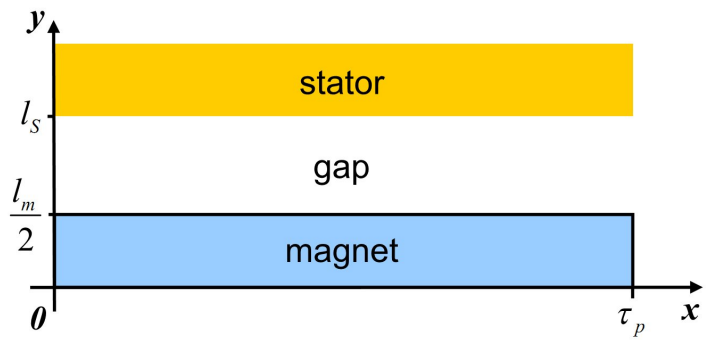

Fig. 5. New reduced geometry with magnetization distribution. The magnetization distribution along the $x$-direction can be expanded into Fourier series

$$
M_{y}=\sum_{n=1}^{\infty} K_{y n} \cdot \cos \left(\frac{n \pi x}{\tau_{p}}\right)
$$

where

$$
K_{y n}=\left\{\begin{array}{cc}
0 & n \text { even } \\
\frac{4}{n \pi} \frac{B_{r}}{\mu_{0}} \sin \left(\frac{n \pi \alpha_{m}}{2}\right) & n \text { odd }
\end{array}\right.
$$

with $B_{r}$ represents the remanence of the magnet and $\alpha_{m}$ is the magnet fraction and is defined as: 


$$
\alpha_{m}=\tau_{m} / \tau_{p}
$$

The magnetostatic field equations for current-free regions are:

$$
\nabla \times \bar{H}=0
$$

and

$$
\nabla \cdot \bar{B}=0
$$

where $\bar{H}$ is the magnetic field strength and $\bar{B}$ is the magnetic flux density. The two fields are coupled by:

$$
\bar{B}=\mu_{0} \bar{H}
$$

in the airgap region and

$$
\bar{B}=\mu_{0} \mu_{r} \bar{H}+\mu_{0} \bar{M}
$$

in the permanent magnet region, where $\bar{M}$ is the magnetization vector $\left(\bar{M}= \pm M_{y} \bar{j}\right), \mu_{r}$ the relative permeability of the magnet and $\mu_{0}=4 \pi \cdot 10^{-7} \mathrm{~T} \mathrm{~m} / \mathrm{A}$ is the permeability of free space. Equation (4) allows us to define a scalar magnetic potential, $v$, such us:

$$
\bar{H}=-\nabla v
$$

Magnetic field strength vector $\bar{H}$ components are deduced from $v$ by:

$$
H_{x}=-\frac{\partial v}{\partial x} \quad H_{y}=-\frac{\partial v}{\partial y}
$$

Substituting equations (8) and (6) or (7) in (5), reduces the magnetostatic Maxwell equation to a Laplace or Poisson equation, given by:

$$
\nabla^{2} v_{I}=0
$$

in the airgap region, and

$$
\nabla^{2} v_{I I}=\frac{\nabla \bar{M}}{\mu_{r}}
$$

in the magnet region. As the magnet poles posses only the $y$-direction magnetization, the divergence of the magnetization vector is zero. Therefore, the scalar potential in both regions, $v_{i}, i=I, I I$, satisfies:

$$
\frac{\partial^{2} v_{i}}{\partial x^{2}}+\frac{\partial^{2} v_{i}}{\partial y^{2}}=0
$$

Solving equation (12) by the method of separation of variables [19], we obtain the general solution in region I:

$$
\begin{gathered}
v_{I}=\sum_{n}\left(A_{n}^{I} \cos \beta_{n}^{I} x \cosh \beta_{n}^{I} y+B_{n}^{I} \cos \beta_{n}^{I} x \sinh \beta_{n}^{I} y+\right. \\
\left.+C_{n}^{I} \sin \beta_{n}^{I} x \cosh \beta_{n}^{I} y+D_{n}^{I} \sin \beta_{n}^{I} x \sinh \beta_{n}^{I} y\right)
\end{gathered}
$$

and in region II:

$$
\begin{aligned}
v_{I I}= & \sum_{n}\left(A_{n}^{I I} \cos \beta_{n}^{I I} x \cosh \beta_{n}^{I I} y+B_{n}^{I I} \cos \beta_{n}^{I I} x \sinh \beta_{n}^{I I} y\right. \\
& \left.+C_{n}^{I I} \sin \beta_{n}^{I I} x \cosh \beta_{n}^{I I} y+D_{n}^{I I} \sin \beta_{n}^{I I} x \sinh \beta_{n}^{I I} y\right)
\end{aligned}
$$

where the constants $A_{n}^{I}, B_{n}^{I}, C_{n}^{I}, D_{n}^{I}, \beta_{n}^{I}, A_{n}^{I I}, B_{n}^{I I}, C_{n}^{I I}$, $D_{n}^{I I}$ and $\beta_{n}^{I I}$ can be determined by imposing appropriate boundary conditions. These boundary condition are:

\section{A. Region I:}

- The normal component of the field is zero along the symmetry line defined by the $y$-axis.

- The normal component of the field is zero along the symmetry line defined by $x=\tau_{p}$.

- The tangential field is zero along the upper boundary defined by $y=l_{s}$.

\section{B. Region II:}

- The normal component of the field is zero along the symmetry line defined by the $y$-axis.

- The normal component of the field is zero along the symmetry line defined by $x=\tau_{p}$.

- The tangential field is zero along the symmetry line defined by $x$-axis.

C. Region I-Region II interface: The normal component of $\bar{B}$ and the tangential component of $\bar{H}$ must be continuous.

Solving the system of equations resulting from applying the boundary conditions detailed above, we determine the constants $A_{n}^{I}, B_{n}^{I}, \ldots . ., D_{n}^{I I}$ and $\beta_{n}^{I I}$, which substituted in the corresponding equations (13) and (14), provide us with the expression of the scalar potential in region I:

$v_{I}=\sum_{n=1}^{\infty} \frac{K_{y n}}{\beta_{n}} \cdot \frac{\sinh \left(\beta_{n} l_{m} / 2\right)}{\sinh \left(\beta_{n} l_{s}\right)} \cdot \cos \left(\beta_{n} x\right) \cdot \sinh \left[\beta_{n}\left(l_{s}-y\right)\right]$

and region II:

$v_{I I}=\sum_{n=1}^{\infty} \frac{K_{y n}}{\beta_{n}} \cdot \frac{\sinh \left(\beta_{n} g\right)}{\sinh \left(\beta_{n} l_{s}\right)} \cdot \cos \left(\beta_{n} x\right) \cdot \sinh \left(\beta_{n} y\right)$

The expressions of the magnetic field strength components and the magnetic flux density components can be obtained using the equations (9) and (6).

In order to verify the expressions obtained by the previous calculations, some 3D FEM simulations have been made with FLUX3D software. The numerical values of the main parameters are given in Table 1. Fig. 6 shows a comparison between the scalar magnetic potential over the magnet surface for the analytical proposed method and the FEM versus the magnet spacer width. It can be seen that there are a very good agreement between the analytical results and simulations obtained with 3D FEM software. We can also observe that in all cases, even when there is no separation between the magnets, the potential on the surface of the magnet changes smoothly between a magnet and another, not being an instantaneous change. The value reaches the scalar potential in the center of the magnet, $V_{0}$, is equal to $3284 A \cdot v$.

Table 1.-Numerical values of the main parameters

\begin{tabular}{lcc}
\hline \hline \multicolumn{1}{c}{ Parameter } & Symbol & Value \\
\hline Magnet remanence & $B_{r}$ & $1.2 \mathrm{~T}$ \\
Magnet recoil permeability & $\mu_{r}$ & 1.05 \\
Magnet axial length & $l_{m}$ & $20 \mathrm{~mm}$ \\
Airgap length & $g$ & $5 \mathrm{~mm}$ \\
Slot opening & $s$ & $7 \mathrm{~mm}$ \\
Teeth width & $t$ & $16 \mathrm{~mm}$ \\
Slot depth & $d_{S}$ & $21.5 \mathrm{~mm}$ \\
Pole pitch & $\tau_{p}$ & $69 \mathrm{~mm}$ \\
Slot pitch & $\tau_{s}$ & $23 \mathrm{~mm}$ \\
\hline
\end{tabular}




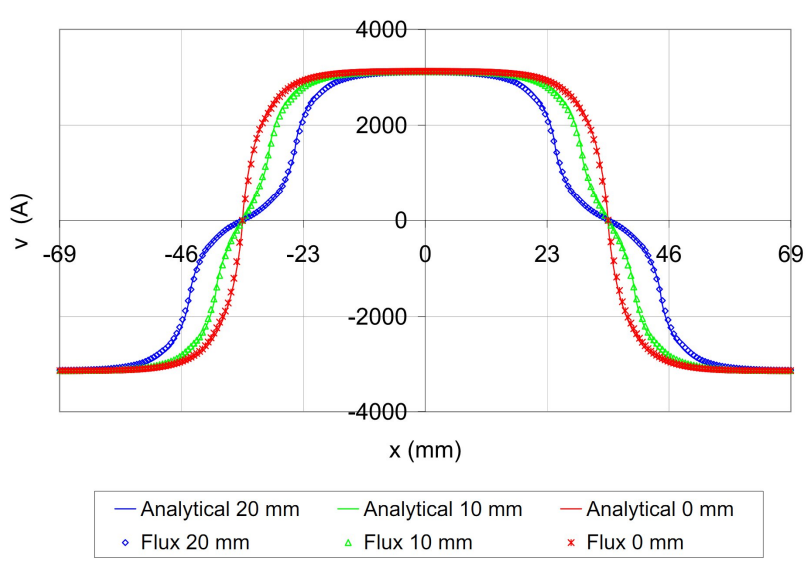

Fig. 6. Comparison between the scalar magnetic potential over the magnet surface for the analytical proposed method and the FEM versus the magnet spacer width.

\section{Slotted machine:}

Once you know the value of the scalar potential on the surface of the magnet, we are able to apply the Fourier series method to the slot machine shown in Figure 1b, in order to obtain the distribution of magnetic scalar potential in the air, ie in the gap and the slots. To apply the method we have to divide the study area into rectangular regions interconnected and, by applying the superposition principle, obtain the potential in each region as a sum of potentials generated by each of its boundary conditions. This requires knowing the potential in all boundaries delimiting each rectangular region.

When we study the slotted machine we have to take into account the relative position of the edge of the magnet on the stator. Therefore, we will distinguish four situations:

- Partial tooth: the edge of the magnet is in front of a tooth of the machine

- Full slot: the edge of the magnet faces the beginning of a slot

- Partial slot: the edge of the magnet is in front of a slot machine

- Full tooth: the edge of the magnet faces the beginning of a tooth

\section{A. Partial tooth:}

In this situation, the edge of the magnet is in front of a tooth of the machine (fig. 7). If we consider a machine pole, we can divide the region under study in seven rectangular regions. Known stator scalar potential $(v=0)$ and the magnet surface scalar potential $\left(v=V_{0}\right)$, we must only know the potential of the common borders to adjacent regions in order to calculate the potential in each of the seven regions. Since two adjacent regions (ie regions III and IV) have a common boundary (IJ), the scalar potential and its spatial derivatives must be continuous across the boundary.

The problem is therefore, solved in two steps [18]:

- Assume an arbitrary potential distribution along the boundaries ( $\mathrm{AB}, \mathrm{CD}, \ldots . \mathrm{VW})$ in terms of some unknown Fourier coefficients. Solve the field equations in each region.

- Obtain the unknown coefficients by matching the normal derivate of the potential function along each boundary $(\mathrm{AB}, \mathrm{CD}, \ldots . \mathrm{VW})$.
Figure 8 shows the corresponding field problem.

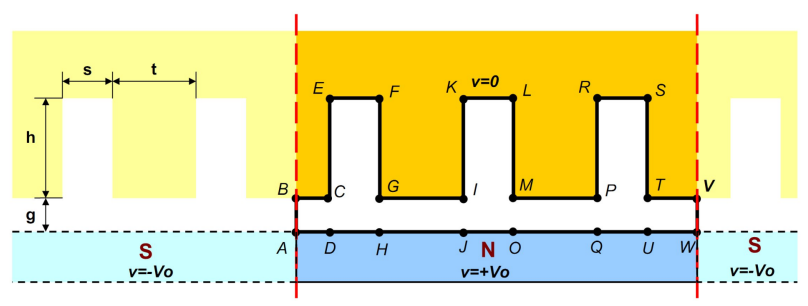

Fig. 7. Studied geometry corresponding to a machine pole for the situation of partial tooth.

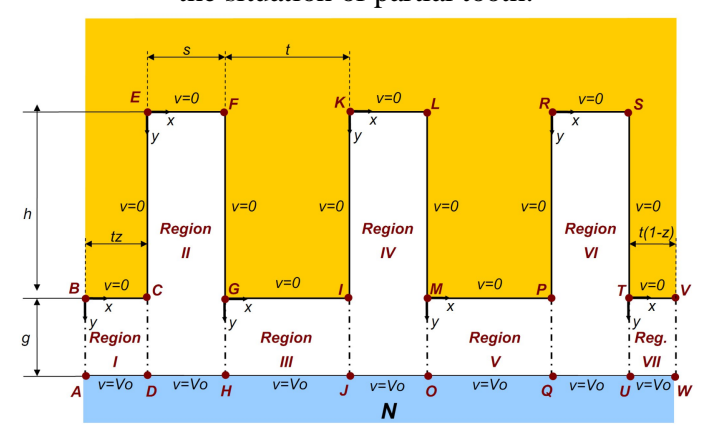

Fig. 8. Field problem corresponding to the geometry shown in fig 7. See Table 1 for the values of the geometric parameters.

As an example, we show the expressions obtained for the scalar potential in the region III. With the $x-y$ coordinates system fixed at $\mathrm{G}$ as shown, let the potential across $G H$ assumed to be given by:

$$
v_{G H}=\sum_{l=1}^{\infty} M_{l} \sin \frac{l \pi y}{g} \quad \text { for } 0 \leq y \leq g
$$

and let the potential across $I J$ assumed to be given by:

$$
v_{I J}=\sum_{m=1}^{\infty} L_{m} \sin \frac{m \pi y}{g} \quad \text { for } 0 \leq y \leq g
$$

being the constants $M_{l}$ and $L_{m}$ the unknown Fourier coefficients. The expression of the magnetic scalar potential in the region III, $v_{I I I}$, results:

$$
\begin{aligned}
v_{I I I} & =\sum_{k=1}^{\infty} \frac{4 \cdot V_{0}}{(2 k-1) \pi} \frac{\sinh \left[\frac{(2 k-1) \pi y}{t}\right]}{\sinh \left[\frac{(2 k-1) \pi g}{t}\right]} \sin \left[\frac{(2 k-1) \pi x}{t}\right]+ \\
& +\sum_{l=1}^{\infty} M_{l} \frac{\sinh \left[\frac{l \pi(t-x)}{g}\right]}{\sinh \left(\frac{l \pi t}{g}\right)} \sin \left(\frac{l \pi y}{g}\right)+ \\
& +\sum_{m=1}^{\infty} L_{m} \frac{\sinh \left(\frac{m \pi x}{g}\right)}{\sinh \left(\frac{m \pi t}{g}\right)} \sin \left(\frac{m \pi y}{g}\right)
\end{aligned}
$$

Following the principle of superposition, the expression of this potential has three terms: the potential due to the magnet and the potential caused by $\mathrm{GH}$ and IJ boundaries.

The expressions of the magnetic flux density components in region III can be obtained using equations (6) and (9).

Applying this procedure to all regions, we get the expression of the scalar potential in each one of them. The determination of the unknown Fourier coefficients is 
performed by matching the normal derivatives of the potential function on both sides of each boundary. Equating these derivatives in a number of values of $y$, we obtain a system of equations whose solution gives us the Fourier coefficients of the potential distributions of each of the boundaries.

The method is applied similarly to the three remaining cases (full slot, partial slot and full tooth).

\section{B. Full slot:}

Figure 9 shows the field problem for the case of full slot. The region corresponding to a pole is divided into 6 rectangular regions interconnected.

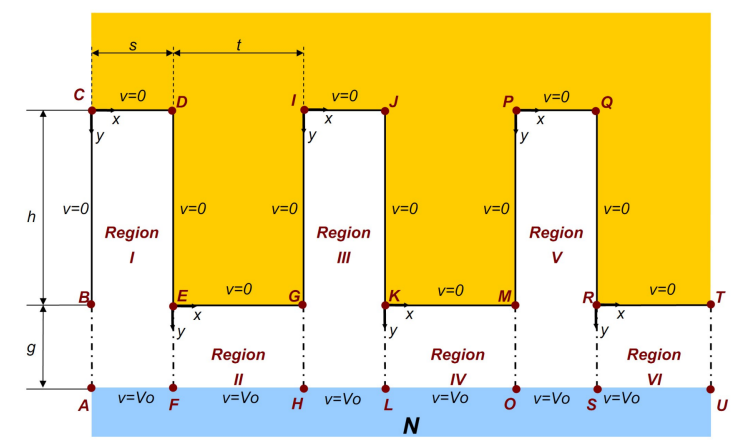

Fig 9. Field problem corresponding to a machine pole for the situation of full slot.

\section{Partial slot:}

Figure 10 shows the field problem for the case of partial slot. The region corresponding to a pole is divided into 7 rectangular regions interconnected.

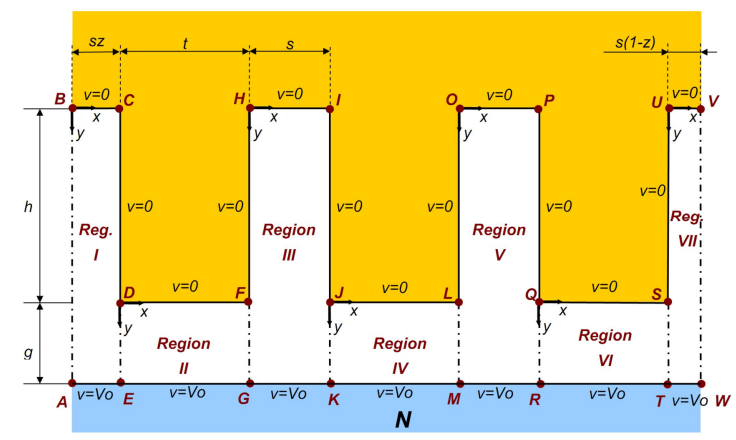

Fig 10. Field problem corresponding to a machine pole for the situation of partial slot.

\section{Full teeth:}

Figure 11 shows the field problem for the case of full teeth. The region corresponding to a pole is divided into 6 rectangular regions interconnected.

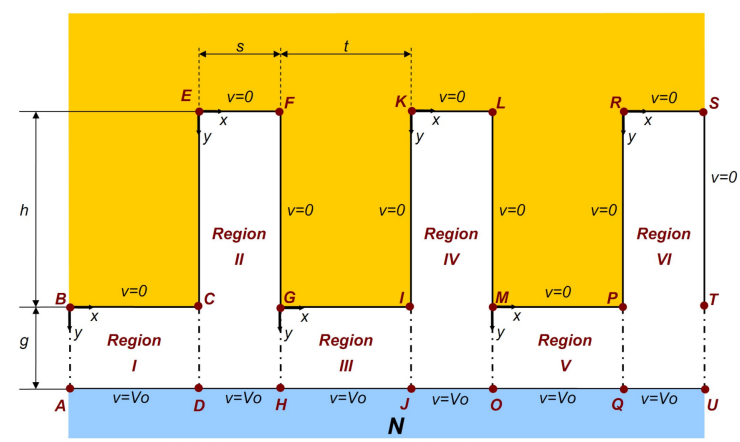

Fig 11. Field problem corresponding to a machine pole for the situation of full teeth.

\section{E. Motion:}

In the field problem for partial slot and partial tooth (Figs. 8 and 10), we have introduced the parameter $z$, which allows us to easily implement motion in the tangential direction. In both figures, if we vary $\mathrm{z}$ from 1 to 0 , the rotor will move to the right (if the movement is to the left, the variation of $\mathrm{z}$ is from 0 to 1 ). We define the offset as the displacement between the initial position (center of the tooth) and the left edge of the magnet. Table 2 shows the offset's expressions for each case. After about a pitch slot $\left(\tau_{s}=t+s\right)$, the field values in the next pitch slot can be deduced from the field values of the preceding pitch slot.

Table 2.- Offset's expressions

\begin{tabular}{llc}
\hline \hline \multicolumn{1}{c}{ Case } & \multicolumn{1}{c}{ Offset } & Range of $\mathrm{z}$ \\
\hline Partial teeth & Offset $=(t / 2)-z \cdot t$ & $0 \leq z<0.5$ \\
Full slot & Offset $=t / 2$ & ------- \\
Partial slot & Offset $=(t / 2)+s(1-z)$ & $0<z<1$ \\
Full teeth & Offset $=(t / 2)+s$ & ------- \\
Partial teeth & Offset $=(t / 2)+s+t(1-z)$ & $0.5<z<1$ \\
\hline
\end{tabular}

\section{Results and Comparison with Finite Element Method Analysis.}

In order to verify the expressions obtained by the previous calculations, some 3D FEM simulations have been made with FLUX3D software. The numerical values of the main parameters are given in Table 1. Fig. 12 shows a comparison between the scalar magnetic potential at the top of the gap, at the middle of the gap and over the magnet surface for the analytical proposed method and the FEM analysis.

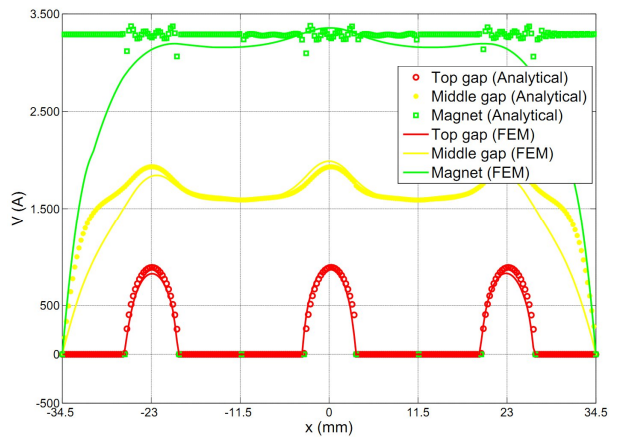

Fig. 12. Magnetic scalar potential at the top of the gap (red), in the middle of the gap (yellow) and over the magnet (green)

Figure 13 compares analytically predicted and finiteelement-calculated open-circuit distributions of the tangential ( $x$ component) and normal (y-component) magnetic flux density components at the top of the gap.

In view of the results shown, it can be seen that there are differences between the values found by analytical and FEM, mainly caused by imposing the potential on the surface of the magnet is uniform and equal to Vo. In section 2, studying the smooth machine, we obtained that the potential on the surface of the magnet in the vicinity of the edges smoothly tended towards zero. To implement this trend, we represent the surface potential of the magnet by hyperbolic tangent function, which will allow us to smoothly reduce the value of Vo in the neighborhood of the edges of the magnet. 


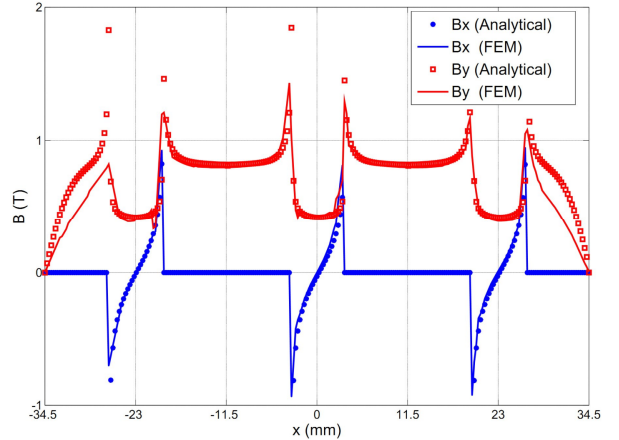

Fig. 13. Magnetic flux density at the top of the gap.

Fig. 14 shows a comparison between the scalar magnetic potential at the top of the gap, at the middle of the gap and over the magnet surface for the analytical proposed method and the FEM analysis using hyperbolic tangent function. Figure 15 compares the analytically and FEM magnetic flux density components at the top of the gap with hyperbolic tangent function.

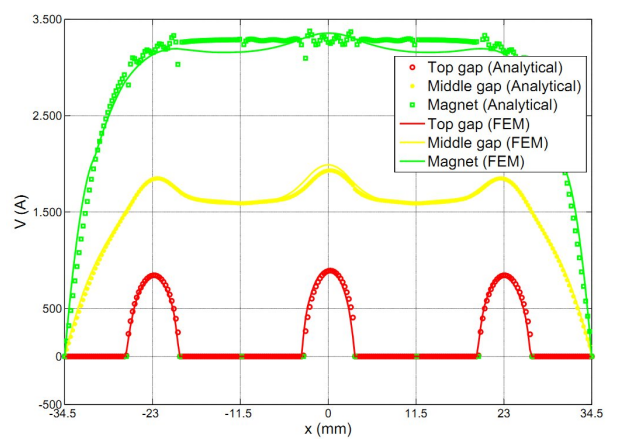

Fig. 14. Magnetic scalar potential with hyperbolic tangent function at the top of the gap (red), in the middle of the gap (yellow) and over the magnet (green)

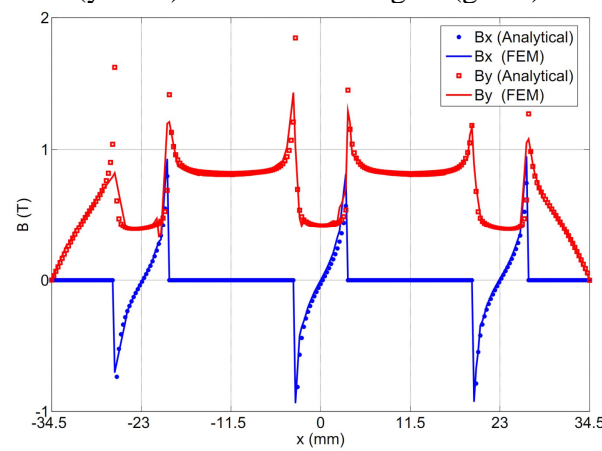

Fig. 15. Magnetic field components at the top of the gap with hyperbolic tangent function.

\section{Conclusion}

A general analytical model for the analysis of an axial flux permanent magnet machine with slots at no load was presented. The proposed method solves the Laplace equation in the gap of an slotted machine via magnetic scalar potential in an interconnected rectangular regions. Having found the scalar potential, we obtain the components of the flux density. The analytical results obtained by the model were compared with those obtained by FEM. The use of the hyperbolic tangent profile allows us to model the potential at the surface of the magnet and improve results. Once the magnetic scalar potential is defined, other parameters, such us the magnetic flux crossing any given area, the induced electromagnetic force or the cogging torque could be computed. In addition to permanent magnet machines, this technique can be applied to any 2D geometry with the restriction that the geometry should consist of rectangular regions.

\section{References}

[1] G. Qishan and G. Hongzhan, "Effect of Slotting in Pm Electric Machines", Electric Machines \& Power Systems, Volume 10, Issue 4, pp. 273-284, 1985

[2] E. P. Furlani, "A method for predicting the field in permanent magnet axial field motors", IEEE Trans. Magn., vol 28, no. 5, pp. 2061-2066, 1992.

[3] Zhu, Z.Q.; Howe, D.; , "Instantaneous magnetic field distribution in brushless permanent magnet DC motors. III. Effect of stator slotting," Magnetics, IEEE Transactions on, vol.29, no.1, pp.143-151, Jan 1993

[4] Liu, Z.J.; Li, J.T.; , "Analytical Solution of Air-Gap Field in Permanent-Magnet Motors Taking Into Account the Effect of Pole Transition Over Slots," Magnetics, IEEE Transactions on, vol.43, no.10, pp.3872-3883, Oct. 2007

[5] Gysen, B.L.J.; Meessen, K.J.; Paulides, J.J.H.; Lomonova, E.A.; , "General Formulation of the Electromagnetic Field Distribution in Machines and Devices Using Fourier Analysis," Magnetics, IEEE Transactions on , vol.46, no.1, pp.39-52, Jan. 2010

[6] Bellara, A.; Amara, Y.; Barakat, G.; Reghem, P.; "Analytical modelling of the magnetic field in axial flux permanent magnet machines with semi-closed slots at no load," Electrical Machines (ICEM), 2010 XIX International Conference on , vol., no., pp.1-6, 6-8 Sept. 2010

[7] N. Boules, "Prediction of no-load flux density distribution in permanent magnet machines" IEEE Trans. Ind. Appl., vol IA-21, no. 4, pp. 663-643, May/June 1985.

[8] J. R. Bumby, R. Martin, M. A. Mueller, E. Spooner, N. L. Brown and B. J. Chalmers, "Electromagnetic design of axial-flux permanent magnet machines", Electric Power Applications, IEE Proceedings, vol. 151, issue 2, pp. 151160, March 2004.

[9] Krop, D.C.J.; Lomonova, E.A.; Vandenput, A.J.A.; , "Application of Schwarz-Christoffel Mapping to Permanent-Magnet Linear Motor Analysis," Magnetics, IEEE Transactions on , vol.44, no.3, pp.352-359, March 2008

[10] Gysen, B.L.J.; Lomonova, E.A.; Paulides, J.J.H.; Vandenput, A.J.A.; , "Analytical and Numerical Techniques for Solving Laplace and Poisson Equations in a Tubular Permanent Magnet Actuator: Part II. SchwarzChristoffel Mapping," Magnetics, IEEE Transactions on , vol.44, no.7, pp.1761-1767, July 2008

[11] Boughrara, K.; Zarko, D.; Ibtiouen, R.; Touhami, O.; Rezzoug, A.; , "Magnetic Field Analysis of Inset and Surface-Mounted Permanent-Magnet Synchronous Motors Using Schwarz-Christoffel Transformation," Magnetics, IEEE Transactions on , vol.45, no.8, pp.3166-3178, Aug. 2009

[12] B. Hague, "The principles of Electromagnetism Applied to Electrical Machines", Dover Publications Inc., New York (1962).

[13] R. H. Engelmann and W. H. Middendorf, "Handbook of electrical motors". Marcel Dekker Inc, New York (1995) pp. 17-20.

[14] R. Haberman, "Ecuaciones en Derivadas Parciales con Series de Fourier y Problemas de Contorno", Prentice Hall (2000).

[15] D. C. Hanselman, "Brushless permanent magnet motor design”. Rhode Island, The Writers' Collective, 2003. 